\title{
AN ODD-POWER IDENTITY INVOLVING DISCRETE CONVOLUTION
}

\author{
PETRO KOLOSOV
}

Abstract. Let be a power function $f_{r, M}(s)$ defined for every $s$ within the finite set $M$ as follows

$$
f_{r, M}(s)= \begin{cases}s^{r}, & s \in M \\ 0, & \text { otherwise }\end{cases}
$$

Let a discrete convolution of $f_{r, M}(s)$ be denoted as follows $\operatorname{Conv}_{r, M}[n]=\left(f_{r, M} * f_{r, M}\right)[n]$. Let a real coefficients $A_{m, j}$ be given by the following recurrence

$$
A_{m, j}:= \begin{cases}0, & \text { if } j<0 \text { or } j>m, \\
(2 j+1)\left(\begin{array}{c}
2 j \\
j
\end{array}\right) \sum_{d=2 j+1}^{m} A_{m, d}\left(\begin{array}{c}
d \\
2 j+1
\end{array}\right) \frac{(-1)^{d-1}}{d-j} B_{2 d-2 j}, & \text { if } 0 \leq j<m, \\
(2 j+1)\left(\begin{array}{c}
2 j \\
j
\end{array}\right), & \text { if } j=m .\end{cases}
$$

In this paper we show that for every $n>0$ the following odd-power identities involving coefficients $A_{m, j}$ and convolution transform $\operatorname{Conv}_{r, M}[n]$ hold

$$
\begin{aligned}
n^{2 m+1}+1 & =\sum_{r=0}^{m} A_{m, r} \operatorname{Conv}_{r, \mathbb{N}}[n], \\
n^{2 m+1}-1 & =\sum_{r=0}^{m} A_{m, r} \operatorname{Conv}_{r, \mathbb{Z}_{>0}}[n], \\
n^{2 m+1} & =\sum_{r=0}^{m} A_{m, r} \sum_{k=1}^{n} k^{r}(n-k)^{r} \\
& =\sum_{r=0}^{m} A_{m, r} \sum_{k=0}^{n-1} k^{r}(n-k)^{r} .
\end{aligned}
$$

\section{Definitions}

- $\mathbb{N}$ - set of natural numbers $\{0,1,2,3, \ldots\}$.

- $\mathbb{Z}_{>0}$ - set of positive integers $\{1,2,3,4, \ldots\}$.

- $\operatorname{Conv}_{r, M}[n]=\left(f_{r, M} * f_{r, M}\right)[n]=\sum_{k} f_{r, M}(k) f_{r, M}(n-k)$ - convolution transform of real function $f_{r, M}(k)$ to itself.

\section{Introduction And Main RESUlts}

The problem of finding expansions of monomials, binomials etc. is classical and there are a lot of beautiful solutions have been found, the most prominent examples are Binomial Theorem [1], Multinomial Theorem [7], Faulhaber's Formula [2], Worpitzky Identity [3], Identity in terms of Stirling numbers of the second kind and falling factorial [4]. Also, the

2010 Mathematics Subject Classification. 11C08 (primary), 44A35 (secondary).

Key words and phrases. Power Identities, Polynomials, Convolution, Convolution power, Integral transforms. 
one good example can be found at [6], so-called MacMillan Double binomial sum. Over decades mathematicians fight against the problem of polynomial expansions and this fight is successful, but still can we find some new approaches to solve this problem? This question is entire motivation of this manuscript. In this paper we solve the classical problem of finding expansions of monomials using convolution transform of power function, which defined on the finite set $M$. Let a power function $f_{r, M}$ be defined as follows

$$
f_{r, M}(s)= \begin{cases}s^{r}, & s \in M, \\ 0, & \text { otherwise. }\end{cases}
$$

Mainly, we assume that the set $M$ is set of natural numbers $\mathbb{N}$ or nonnegative integers $\mathbb{Z}_{>0}$. By this assumption it follows that convolution of $f_{r, M}(s)$ has a discrete form. Let the discrete convolution of $f_{r, M}(s)$ be defined as follows

$$
\operatorname{Conv}_{r, M}[n]:=\left(f_{r, M} * f_{r, M}\right)[n]=\sum_{k} f_{r, M}(k) f_{r, M}(n-k) .
$$

If $M$ is subset, but not a proper subset of $\mathbb{N}$ or $\mathbb{Z}_{>0}$, the formula (2.2) reduces to

$$
\operatorname{Conv}_{r, M}[n]= \begin{cases}\sum_{k=0}^{n} k^{r}(n-k)^{r}, & \text { if } M \subseteq \mathbb{N}, \\ \sum_{k=1}^{n-1} k^{r}(n-k)^{r}, & \text { if } M \subseteq \mathbb{Z}_{>0} .\end{cases}
$$

Property 2.3. For every $n, k$

$$
f_{r, M}(k) f_{r, M}(n-k)=f_{r, M}(n-k) f_{r, M}(n-(n-k))=f_{r, M}(n-k) f_{r, M}(k) .
$$

Let a real coefficients $A_{m, j}$ be defined by the following recurrence relation

\section{Proposition 2.4.}

$$
A_{m, j}:= \begin{cases}0, & \text { if } j<0 \text { or } j>m, \\
(2 j+1)\left(\begin{array}{c}
2 j \\
j
\end{array}\right) \sum_{d=2 j+1}^{m} A_{m, d}\left(\begin{array}{c}
d \\
2 j+1
\end{array}\right) \frac{(-1)^{d-1}}{d-j} B_{2 d-2 j}, & \text { if } 0 \leq j<m, \\
(2 j+1)\left(\begin{array}{c}
2 j \\
j
\end{array}\right), & \text { if } j=m .\end{cases}
$$

Example of coefficients $A_{m, j}$ arranged in table

\begin{tabular}{c|cccccc}
$m / r$ & 0 & 1 & 2 & 3 & 4 & 5 \\
\hline 0 & 1 & & & & & \\
1 & 1 & 6 & & & & \\
2 & 1 & 0 & 30 & & & \\
3 & 1 & -14 & 0 & 140 & & \\
4 & 1 & 120 & 0 & 0 & 630 & \\
5 & 1 & -1386 & 660 & 0 & 0 & 2772
\end{tabular}

Table 1. Coefficients $A_{m, r}$.

Note that the set of $A_{m, j}$ consists fractions for $m \geq 11$. As Table 1 shows, for every $m$, the $A_{m, 0}=1$. 
The following theorem shows the odd-power identity involving coefficients $A_{m, j}$ and convolution transform $\operatorname{Conv}_{r, M}[n]=\left(f_{r, M} * f_{r, M}\right)[n]$

Theorem 2.5. For every $n, m \in \mathbb{N}$

$$
n^{2 m+1}+1=\sum_{r=0}^{m} A_{m, r} \operatorname{Conv}_{r, \mathbb{N}}[n]=\sum_{r=0}^{m} A_{m, r} \sum_{k=0}^{n} k^{r}(n-k)^{r}, n>0 .
$$

As $k$ approaches $n$ in the sum $\sum_{k=0}^{n} k^{r}(n-k)^{r}$, the $k^{r}(n-k)^{r}$ takes nonzero value only in case when $r=0$,

$$
k^{r}(n-k)^{r}= \begin{cases}1, & \text { if } k=n, r=0 \\ 0, & \text { if } k=n, r>0\end{cases}
$$

we assume that there is $0^{0}=1$ in (2.6). By the (2.6) and Theorem 2.5,

Corollary 2.7. For every $n, m \in \mathbb{N}$

$$
n^{2 m+1}-1=\sum_{r=0}^{m} A_{m, r} \operatorname{Conv}_{r, \mathbb{Z}}>0
$$

Corollary 2.8. For every $n, m \in \mathbb{N}$

$$
n^{2 m+1}=\sum_{r=0}^{m} A_{m, r} \sum_{k=0}^{n-1} k^{r}(n-k)^{r}=\sum_{k=0}^{n-1} \sum_{r=0}^{m} A_{m, r} k^{r}(n-k)^{r} .
$$

By the Property 2.3 (symmetry of $k^{r}(n-k)^{r}$ ), we also can rewrite Corollary 2.8 as

$$
n^{2 m+1}=\sum_{k=0}^{n-1} \sum_{r=0}^{m} A_{m, r} k^{r}(n-k)^{r}=\sum_{k=1}^{n} \sum_{r=0}^{m} A_{m, r} k^{r}(n-k)^{r} .
$$

One another interesting observation concerning the coefficients $A_{m, r}$, the sum of $A_{m, r}$ over $r$ gives

$$
\sum_{r=0}^{m} A_{m, r}=2^{2 m+1}-1
$$

Expression (2.9) is partial case of Corollary 2.8 for $n=2$, it works since the for every $r$, the $\operatorname{Conv}_{r, \mathbb{Z}_{>0}}[2]=1$. 


\section{Proof of Theorem 2.5}

Proof. By the Corollary 2.8, the coefficients $A_{m, r}$ could be evaluated expanding $\sum_{k=0}^{n-1} k^{r}(n-$ $k)^{r}$ and using Faulhaber's formula $\sum_{k=1}^{n} k^{p}=\frac{1}{p+1} \sum_{j=0}^{p}\left(\begin{array}{c}p+1 \\ j\end{array}\right) B_{j} n^{p+1-j}$, we get

$$
\begin{aligned}
& \sum_{k=0}^{n-1} k^{r}(n-k)^{r} \\
& =\sum_{k=0}^{n-1} k^{r} \sum_{j}(-1)^{j}\left(\begin{array}{l}
r \\
j
\end{array}\right) n^{r-j} k^{j}=\sum_{j}(-1)^{j}\left(\begin{array}{l}
r \\
j
\end{array}\right) n^{r-j}\left(\sum_{k=0}^{n-1} k^{r+j}\right) \\
& =\sum_{j}\left(\begin{array}{l}
r \\
j
\end{array}\right) n^{r-j} \frac{(-1)^{j}}{r+j+1}\left[\sum_{s}\left(\begin{array}{c}
r+j+1 \\
s
\end{array}\right) B_{s} n^{r+j+1-s}-B_{r+j+1}\right] \\
& =\sum_{j, s}\left(\begin{array}{l}
r \\
j
\end{array}\right) \frac{(-1)^{j}}{r+j+1}\left(\begin{array}{c}
r+j+1 \\
s
\end{array}\right) B_{s} n^{2 r+1-s}-\sum_{j}\left(\begin{array}{c}
r \\
j
\end{array}\right) \frac{(-1)^{j}}{r+j+1} B_{r+j+1} n^{r-j} \\
& =\sum_{s} \underbrace{\sum_{j}\left(\begin{array}{l}
r \\
j
\end{array}\right) \frac{(-1)^{j}}{r+j+1}\left(\begin{array}{c}
r+j+1 \\
s
\end{array}\right)}_{S(r)} B_{s} n^{2 r+1-s}-\sum_{j}\left(\begin{array}{l}
r \\
j
\end{array}\right) \frac{(-1)^{j}}{r+j+1} B_{r+j+1} n^{r-j}
\end{aligned}
$$

where $B_{s}$ are Bernoulli numbers and $B_{1}=\frac{1}{2}$. Now, we notice that

$$
S(r)=\sum_{j}\left(\begin{array}{l}
r \\
j
\end{array}\right) \frac{(-1)^{j}}{r+j+1}\left(\begin{array}{c}
r+j+1 \\
s
\end{array}\right)= \begin{cases}\frac{1}{(2 r+1)\left(\begin{array}{c}
2 r \\
r
\end{array}\right)}, & \text { if } s=0 \\
\frac{(-1)^{r}}{s}\left(\begin{array}{r}
r \\
2 r-s+1
\end{array}\right), & \text { if } s>0 .\end{cases}
$$

In particular, the last sum is zero for $0<s \leq r$. Therefore, expression (3.1) takes the form

$$
\begin{aligned}
\sum_{k=0}^{n-1} k^{r}(n-k)^{r} & =\frac{1}{(2 r+1)\left(\begin{array}{c}
2 r \\
r
\end{array}\right)} n^{2 r+1}+\underbrace{\sum_{s \geq 1} \frac{(-1)^{r}}{s}\left(\begin{array}{c}
r \\
2 r-s+1
\end{array}\right) B_{s} n^{2 r+1-s}}_{(\star)} \\
& -\underbrace{\sum_{j}\left(\begin{array}{c}
r \\
j
\end{array}\right) \frac{(-1)^{j}}{r+j+1} B_{r+j+1} n^{r-j}}_{(\diamond)}
\end{aligned}
$$

Hence, introducing $\ell=2 r+1-s$ to $(\star)$ and $\ell=r-j$ to $(\diamond)$, we get

$$
\begin{aligned}
\sum_{k=0}^{n-1} k^{r}(n-k)^{r} & =\frac{1}{(2 r+1)\left(\begin{array}{c}
2 r \\
r
\end{array}\right)} n^{2 r+1}+\sum_{\ell} \frac{(-1)^{r}}{2 r+1-\ell}\left(\begin{array}{l}
r \\
\ell
\end{array}\right) B_{2 r+1-\ell} n^{\ell} \\
& -\sum_{\ell}\left(\begin{array}{c}
r \\
\ell
\end{array}\right) \frac{(-1)^{j-\ell}}{2 r+1-\ell} B_{2 r+1-\ell} n^{\ell} \\
& =\frac{1}{(2 r+1)\left(\begin{array}{c}
2 r \\
r
\end{array}\right)} n^{2 r+1}+2 \sum_{\text {odd } \ell} \frac{(-1)^{r}}{2 r+1-\ell}\left(\begin{array}{c}
r \\
\ell
\end{array}\right) B_{2 r+1-\ell} n^{\ell}
\end{aligned}
$$


Using the definition of $A_{m, r}$ coefficients, we obtain the following identity for polynomials in $n$

$$
\sum_{r} A_{m, r} \frac{1}{(2 r+1)\left(\begin{array}{c}
2 r \\
r
\end{array}\right)} n^{2 r+1}+2 \sum_{r, \text { odd } \ell} A_{m, r} \frac{(-1)^{r}}{2 r+1-\ell}\left(\begin{array}{l}
r \\
\ell
\end{array}\right) B_{2 r+1-\ell} n^{\ell} \equiv n^{2 m+1}
$$

Taking the coefficient of $n^{2 m+1}$ in $(3.2)$ we get $A_{m, m}=(2 m+1)\left(\begin{array}{c}2 m \\ m\end{array}\right)$ and taking the coefficient of $n^{2 d+1}$ for an integer $d$ in the range $m / 2 \leq d<m$, we get $A_{m, d}=0$. Taking the coefficient of $n^{2 d+1}$ for $d$ in the range $m / 4 \leq d<m / 2$, we get

i.e,

$$
A_{m, d} \frac{1}{(2 d+1)\left(\begin{array}{c}
2 d \\
d
\end{array}\right)}+2(2 m+1)\left(\begin{array}{c}
2 m \\
m
\end{array}\right)\left(\begin{array}{c}
m \\
2 d+1
\end{array}\right) \frac{(-1)^{m}}{2 m-2 d} B_{2 m-2 d}=0
$$

$$
A_{m, d}=(-1)^{m-1} \frac{(2 m+1) !}{d ! d ! m !(m-2 d-1) !} \frac{1}{m-d} B_{2 m-2 d} .
$$

Continue similarly, we can express $A_{m, d}$ for each integer $d$ in range $m / 2^{s+1} \leq d<m / 2^{s}$ (iterating consecutively $s=1,2 \ldots$ ) via previously determined values of $A_{m, j}$ as follows

$$
A_{m, d}=(2 d+1)\left(\begin{array}{c}
2 d \\
d
\end{array}\right) \sum_{j \geq 2 d+1} A_{m, j}\left(\begin{array}{c}
j \\
2 d+1
\end{array}\right) \frac{(-1)^{j-1}}{j-d} B_{2 j-2 d}
$$

Thus, for every $(n, m) \in \mathbb{N}$ holds

$$
n^{2 m+1}=\sum_{r=0}^{m} A_{m, r} \sum_{k=0}^{n-1} k^{r}(n-k)^{r}
$$

By the (2.6), for every $k=0$ or $k=n$ in the convolution $\operatorname{Conv}_{r, \mathbb{N}}[n]=\sum_{k=0}^{n} k^{r}(n-k)^{r}$, the term $k^{r}(n-k)^{r}$ equals to

$$
k^{r}(n-k)^{r}= \begin{cases}1, & \text { if } k=n, r=0 \\ 0, & \text { if } k=n, r>0\end{cases}
$$

Thus, Theorem 2.5, holds for every natural $n>0$. This completes the proof.

\section{Acknowledgements}

We would like to thank to Dr. Max Alekseyev (Department of Mathematics and Computational Biology, George Washington University) for sufficient help in the derivation of $A_{m, j}$ coefficients, Also, we'd like to thank to OEIS editors Michel Marcus, Peter Luschny, Jon E. Schoenfield and others for their patient, faithful volunteer work and for useful comments and suggestions during the editing of sequences, concerned with this manuscript.

\section{REFERENCES}

[1] Abramowitz, M. and Stegun, I. A. (Eds.). Handbook of Mathematical Functions with Formulas, Graphs, and Mathematical Tables, 9th printing. New York: Dover, pp. 10, 1972.

[2] Knuth, D. E., Johann Faulhaber and Sums of Powers, pp. 9-10., arXiv preprint, arXiv:math/9207222v1 [math.CA], 1992.

[3] Worpitzky, J.. Studien uber die Bernoullischen und Eulerschen Zahlen.. Journal fur die reine und angewandte Mathematik 94 (1883): 203-232. http://eudml.org/doc/148532.

[4] Graham, Ronald, Knuth Donald, and Oren Patashnik (1989-01-05). Special Numbers. Concrete Mathematics (2nd ed.). Addison Wesley Longman Publishing Co. p. 262. ISBN 0-201-14236-8. 
[5] D. V. Widder et al, "The Convolution Transform.", Bull. Amer. Math. Soc. 60 (1954), 444-456. https://doi.org/10.1090/S0002-9904-1954-09828-2.

[6] Weisstein, Eric W. "Power." From MathWorld-A Wolfram Web Resource, equation 12.

[7] Abramowitz, M. and Stegun, I. A. (Eds.). "Multinomial Coefficients." 24.1.2 in Handbook of Mathematical Functions with Formulas, Graphs, and Mathematical Tables, 9th printing. New York: Dover, pp. 823-824, 1972.

E-mail address: kolosovp94@gmail.com

$U R L:$ https://kolosovpetro.github.io 